\title{
MODIFICATION OF NEUROBEHAVIORAL EFFCTS OF MERCURY BY A GENETIC POLYMORPHISM OF COPROPORPHYRINOGEN OXIDASE IN CHILDREN
}

\author{
James S. Woods ${ }^{\mathrm{a}}$, Nicholas J. Heyer ${ }^{\mathrm{b}}$, Diana Echeverria ${ }^{\mathrm{a}}$, Joan E. Russo ${ }^{\mathrm{c}}$, Michael D. \\ Martin $^{\mathrm{d}}$, Mario F. Bernardo ${ }^{\mathrm{e}}$, Henrique S. Luis ${ }^{\mathrm{e}}$, Lurdes Vaz $^{\mathrm{e}}$, and Federico M. Farin ${ }^{\mathrm{a}}$ \\ aDepartment of Environmental and Occupational Health Sciences, University of Washington, \\ Seattle, Washington, USA \\ bBattelle Centers for Public Health Research and Evaluation, Seattle Washington, USA \\ 'Department of Psychiatry and Behavioral Sciences, University of Washington, Seattle, \\ Washington, USA \\ ${ }^{\mathrm{d} D e p a r t m e n t}$ of Oral Medicine, University of Washington, Seattle, Washington, USA \\ eFaculdade de Medicina Dentaria, Universidade de Lisboa, Lisbon, Portugal
}

\begin{abstract}
Mercury (Hg) is neurotoxic, and children may be particularly susceptible to this effect. A current major challenge is the identification of children who may be uniquely susceptible to $\mathrm{Hg}$ toxicity because of genetic disposition. We examined the hypothesis that CPOX4, a genetic variant of the heme pathway enzyme coproporphyrinogen oxidase (CPOX) that affects susceptibility to mercury toxicity in adults, also modifies the neurotoxic effects of $\mathrm{Hg}$ in children. Five hundred seven children, 8-12 years of age at baseline, participated in a clinical trial to evaluate the neurobehavioral effects of $\mathrm{Hg}$ from dental amalgam tooth fillings in children. Subjects were evaluated at baseline and at 7 subsequent annual intervals for neurobehavioral performance and urinary mercury levels. Following the completion of the clinical trial, genotyping assays for CPOX4 allelic status were performed on biological samples provided by 330 of the trial participants. Regression modeling strategies were employed to evaluate associations between CPOX4 status, Hg exposure, and neurobehavioral test outcomes. Among girls, few significant CPOX4-Hg interactions or independent main effects for Hg or CPOX4 were observed. In contrast, among boys, numerous significant interaction effects between CPOX4 and $\mathrm{Hg}$ were observed spanning all 5 domains of neurobehavioral performance. All underlying dose-response associations between $\mathrm{Hg}$ exposure and test performance were restricted to boys with the CPOX4 variant, and all of these associations were in the expected direction where increased exposure to $\mathrm{Hg}$ decreased performance. These findings are the first to demonstrate genetic susceptibility to the adverse neurobehavioral effects of $\mathrm{Hg}$ exposure in children. The paucity of responses among
\end{abstract}

(C) 2012 Elsevier Inc. All rights reserved.

Correspondence concerning this article should be addressed to: James S. Woods, Ph.D., M.P.H., Department of Environmental and Occupational Health Sciences, University of Washington, 4225 Roosevelt Way NE, Suite 100, Seattle, WA, USA 98105, Telephone: 206 685-3443; Fax: 206 685-4696,jwoods@u.washington.edu.

Publisher's Disclaimer: This is a PDF file of an unedited manuscript that has been accepted for publication. As a service to our customers we are providing this early version of the manuscript. The manuscript will undergo copyediting, typesetting, and review of the resulting proof before it is published in its final citable form. Please note that during the production process errors may be discovered which could affect the content, and all legal disclaimers that apply to the journal pertain.

Conflict of Interest Statement

The authors declare that there are no conflicts of interest. 
same-age girls with comparable $\mathrm{Hg}$ exposure provides evidence of sexual dimorphism in genetic susceptibility to the adverse neurobehavioral effects of $\mathrm{Hg}$ in children and adolescents.

\section{Keywords}

mercury; behavior; neurotoxicity; genetic polymorphism; CPOX4; children

\section{Introduction}

Children are recognized as having heightened susceptibility to the adverse effects of environmental chemicals, as compared with adults with similar exposures (Faustman et al. 2000; Landrigan and Goldman 2011). Of particular concern in this respect are possible neurological deficits associated with mercury exposure (Clarkson 2003; Echeverria et al. 1998; Goering et al. 1992), which may cause impairment of the developing central nervous system along with attendant personality, cognitive function and behavioral disorders (Counter and Buchanan 2004; Davidson et al. 2004; Levy et al. 2004). A current major challenge is the identification of those children who may be uniquely susceptible to $\mathrm{Hg}$ mediated neurological deficits because of genetic predisposition.

Previous studies in adults have identified at least 4 commonly expressed genetic polymorphisms that modify the effects of $\mathrm{Hg}$ on a wide range of neurobehavioral functions (Echeverria et al. 2005, 2006, 2010; Heyer et al. 2004, 2008, 2009). Of particular interest in this respect is a single nucleotide polymorphism $(\mathrm{A}>\mathrm{C})(\mathrm{rs} 1131857)$ in exon 4 of the gene encoding an asparagine-to-histidine change at amino acid $272(\mathrm{~N} 272 \mathrm{H})$ of the heme biosynthetic pathway enzyme, coproporphyrinogen oxidase (CPOX, EC 1.3.3.3). This variant, referred to herein as "CPOX4", both increases sensitivity to the neurobehavioral effects of $\mathrm{Hg}$ (Echeverria et al. 2006) and modifies urinary porphyrin excretion as a potential biomarker of this effect (Woods et al. 2005; Li and Woods 2009). The population frequencies of the homozygous wildtype (A/A), heterozygous (A/C) and homozygous mutant $(\mathrm{C} / \mathrm{C})$ genotypes within this cohort were $0.72,0.25$, and 0.03 , respectively, and were equally prevalent among males and females, suggesting substantial exposure to the CPOX4 variant.

In the present study, we examined the hypothesis that CPOX4 would modify the adverse neurobehavioral effects of $\mathrm{Hg}$ exposure in children as previously observed in adults. Subjects were children and adolescents who participated in a recently completed prospective randomized dental amalgam clinical trial between ages 8-18 and for whom longitudinal (annual) neurobehavioral assessments and quantitative measures of dental amalgam $\mathrm{Hg}$ exposure over 7 years of follow-up were available. Additionally, to preclude selection bias possibly associated with those genotyped for CPOX4 per se, we made comparable assessments with respect to second single nucleotide polymorphism located at exon 5 (rs1729995) (G>A) of the CPOX gene encoding a synonymous mutation in the CPOX enzyme (E330E), referred to herein as "CPOX5". CPOX5 has been previously identified as distributed among men and women within our adult dental population with frequencies of the homozygous common (wildtype), heterozygous, and homozygous mutant alleles of 0.48 , 0.43 and 0.09 , respectively (Woods et al., 2005). CPOX5 is not known to be in linkage disequilibrium with CPOX4. We made these assessments independently in boys and girls. 


\section{Methods}

\subsection{The Study Population}

The current study was performed on a subset of 330 subjects who participated as children in the Casa Pia Dental Amalgam Clinical Trial (DeRouen et al. 2002; 2006) conducted between 1996 and 2006. Participants in the clinical trial included 279 boys and 228 girls, aged 8-12 yrs at baseline, who were students of the Casa Pia school system in Lisbon, Portugal. Children were initially randomized to $\mathrm{Hg}$ amalgam (treatment) or composite resin (control) dental treatment groups. Subjects were evaluated at baseline and at 7 subsequent annual intervals following initial dental treatment using an extensive battery of neurobehavioral assessments (Slade et al. 2008; Townes et al. 2008a, 2008b). Follow-up data were obtained on a similar number of subjects in each treatment group. Studies conducted during the course of the clinical trial (Evens et al. 2001) demonstrated that the children had no significant exposure to methylmercury from dietary fish consumption. However, other unidentified sources of environmental mercury exposure may have contributed to baseline urinary mercury concentrations, which were $1.5 \pm 1.2(0.1-7.7)$ and $1.4 \pm 1.1(0.0-8.6) \mu \mathrm{g} / \mathrm{L}$ for amalgam and composite groups, respectively. Mean urinary mercury concentrations by treatment group and by gender for each year of the clinical trial have been previously published (Woods et al. 2007).

\subsection{Neurobehavioral tests employed}

A comprehensive neurobehavioral test battery was used in this analysis, including measures from the Rays Verbal Learning Test (RAVALT), subtests from the Wide Range Assessment of Memory and Learning and Visual Motor Abilities (WRAVMA), the Wechsler Intelligence Scale for Children III (WISC-III), and the Wechsler Intelligence Scale for Adults-III (WMS-III), Simple Reaction Time, Finger tapping, Trailmaking A and B, the Stroop test, and Wisconsin Card Sort. The validity and rationale underlying the use of these tests in the clinical trial have been described (Martins et al. 2005).

Table 1 lists the 23 neurobehavioral tests that were assessed and presents their means and standard deviations at their last year of administration (Year 7). Tests are organized within the behavioral domains that were evaluated in the clinical trial (DeRouen et al., 2006). Arrows depict whether the test score increases or decreases in magnitude with improved performance. Diminished or adversely affected performance associated with $\mathrm{Hg}$ exposure or CPOX4 variant status is described as occurring in the direction of impaired performance, whereas enhanced or beneficially affected performance associated with either of these variables is described as occurring in the direction of improved performance. The Comprehensive Test Of Nonverbal Intelligence (CTONI) was given to each child at the beginning of the clinical trial to obtain a measure of IQ at baseline.

\subsection{Genotyping assays}

Genotyping for the present study was performed on DNA extracted from buccal cell samples that were obtained from study subjects following completion of the clinical trial $(n=199)$ or from blood samples that were obtained at baseline for blood lead assessments $(n=152)$. Genotyping was performed by the Functional Genomics Laboratory of the NIEHS Center for Ecogenetics and Environmental Health at the University of Washington, using automated DNA sequencing assays. Oligonucleotides used for PCR and sequencing as well as primer and allele-specific probes used for fluorescent 5'-nuclease assays have been previously described in detail (Woods et al. 2005). In the present study, each child was evaluated for CPOX4 gene status, and categorized as CPOX wild type (A/A) or CPOX4 (A/ $\mathrm{C}$ or $\mathrm{C} / \mathrm{C}$ ) if either a single or double allelic variant, respectively, was found. Children were 
also characterized for the CPOX5 variant as CPOX wildtype (G/G) or CPOX5 (G/A or A/A) if either a single or double allelic variant, respectively, was found.

\subsection{Human subjects considerations}

All parents or guardians of children who participated in the clinical trial gave written consent, and all children provided signed assent, for the treatments and assessments made during the course of the trial, including collection of blood samples. Written consent was also obtained from all participants who provided buccal cell samples for genotyping subsequent to completion of the clinical trial. The study protocols for both the clinical trial and the present genotyping study were approved by the institutional review boards at the University of Lisbon and the University of Washington.

\subsection{Urinary mercury analysis}

A urine sample $(\sim 50 \mathrm{ml})$ was collected from each child at baseline of the clinical trial and at each subsequently scheduled annual visit to the University of Lisbon School of Dental Medicine for dental, neurologic, and neurobehavioral evaluations. Analysis of total mercury (Hg) was performed by continuous flow, cold vapor spectrofluorometry, as previously described (Pingree et al. 2001). Urinary creatinine concentrations were measured using a standard colorimetric procedure (Sigma \#555-A; Sigma-Aldrich, St. Louis, MO, USA). Urinary $\mathrm{Hg}$ concentrations were calculated as micrograms per gram creatinine $(\mu \mathrm{g} / \mathrm{g}$ creatinine). Urinary $\mathrm{Hg}$ concentrations were transformed into natural logs after adding one $[\ln (\mathrm{HgU}+1)]$ and used in this form as a quantitative measure of $\mathrm{Hg}$ exposure for all statistical analyses.

\subsection{Statistical Analyses}

This study evaluated whether CPOX4 or CPOX5 gene status affected the relationship between $\mathrm{Hg}$ exposure and tests of neurobehavioral functions among children who were evaluated annually from baseline through 7 years of follow-up after initial placement of dental amalgam (Hg) or composite resin tooth fillings (DeRouen et al. 2006).

Urinary $\mathrm{Hg}$ concentrations $(\mathrm{HgU})$ measured at each annual behavioral test session were employed as the measure of $\mathrm{Hg}$ exposure instead of the dichotomous assignment to amalgam or composite resin treatment groups as performed in the clinical trial. Treatment assignment from the clinical trial was evenly split among boys ( 81 from the composite group and 83 from the amalgam group), whereas girls came slightly more frequently from the amalgam group (74 composite and 92 amalgam). However, treatment assignment accounted, at most, for only $17 \%$ of the variation in $\mathrm{HgU}$ among boys (Year $2 \mathrm{r}^{2}=0.171$ ) and $15 \%$ among girls (Year $2 \mathrm{r}^{2}=0.154$ ).

Statistical analyses were performed using SPSS Version 19 (IBM®SPSS ${ }^{\circ}$, Chicago, IL, USA). The wide range in ages of subjects at the beginning of the clinical trial (8-12 years) and the changing of specific tests administered to various age groups during the course of the trial, e.g., child versus adult versions of some tests, militated against the propitious use of repeated measures analysis. Therefore, we employed concurrent urinary $\mathrm{Hg}$ concentrations $(\mathrm{HgU})$ and neurobehavioral test performance data acquired from the second year of follow-up (Year 2, where mean $\mathrm{HgU}$ reached a peak among both boys and girls in the cohort) to estimate the acute effects of $\mathrm{Hg}$ exposure on performance, and both maximum and cumulative measures of $\mathrm{HgU}$ over the entire study period as well as performance outcomes during the last year of the study (Year 7) to evaluate chronic effects of $\mathrm{Hg}$ exposure. 
Acute measures of $\mathrm{Hg}$ exposure were calculated as the natural $\log$ of $\mathrm{HgU}$ adjusted by 1 $(\ln [\mathrm{HgU}+1])$. The natural log best accommodates how exposures are distributed biologically, whereas adding 1 minimizes the influence of very small changes in $\mathrm{HgU}$ at very low levels (which could have otherwise dominated the analyses). Maximum $\mathrm{HgU}$ was simply the largest value of this acute measure across all study years. Cumulative $\mathrm{HgU}$ was calculated as the natural $\log$ of the sum of all $\mathrm{HgU}$ with 1 added to this summation $(\ln [(\mathrm{LHgU})+1])$.

Because the effect of the CPOX4 variant on neurobehavioral performance as affected by $\mathrm{Hg}$ exposure was the principal focus of this study, we developed an analytical protocol reflecting this focus. As a first step, we used a base model that included the measure of $\mathrm{Hg}$ exposure (as defined above), CPOX allelic status (dichotomous as either wildtype or het/mut for CPOX4), and their interaction term. In addition, this model included the covariates of age at assessment, race, and non-verbal IQ (determined at baseline). Performances on neurobehavioral tests were each individually evaluated as the outcome variable in separate analyses for boys and girls.

Step two was initiated whenever there was a significant interaction term between $\mathrm{Hg}$ exposure and CPOX allelic status. This finding was taken as evidence of effect modification, and thus analyses of effect between $\mathrm{Hg}$ exposure and test performance were calculated separately among boys or girls genotyped as either CPOX wildtype or CPOX4 variant status. This strategy provided a clear description of $\mathrm{Hg}$ dose-response relationships within each genotypic group.

Step three involved evaluating main effects when the interaction term between $\mathrm{Hg}$ exposure and CPOX allelic status when genotyped for CPOX4, was not significant. In this case, the interaction term was dropped from the baseline model, and the main effects of $\mathrm{Hg}$ exposure and CPOX status were evaluated among the full cohort of boys or girls.

This analytical approach was repeated for children genotyped for CPOX5 allelic status.

Prior to fitting the regression models, we examined the assumptions of the model by scrutinizing the distributions and variances of all cognitive tests and $\ln (\mathrm{Hg})$. Most distributions had no significant deviation from normality or inflated variance. After fitting each model, we examined the standardized residuals for statistical outliers. In the rare event that an outlier was found, it was removed and the model was refit.

\section{Results}

The study cohort consisted of 330 children (164 boys and 166 girls) for whom CPOX4 or CPOX5 gene status was available from among 507 total subjects enrolled at the start of the clinical trial. Excluded subjects either did not provide a blood sample at the initiation of the study, or were lost to follow-up for acquisition of a buccal cell sample following completion of the trial. Table 2 presents the characteristics of the cohort at Entry as well as at Years 2 and 7 of the clinical trial. Both boys and girls averaged 10.1 years of age, and most were in the $4^{\text {th }}$ grade at entry into the study. Approximately $74 \%$ of boys and $71 \%$ girls were Caucasian at Entry, and each had an average non-verbal IQ score of 86. Table 2 also displays the mean "Raw" values for urinary $\mathrm{Hg}$ concentrations $(\mathrm{HgU})$ and the natural $\mathrm{log}$ calculations for the $\mathrm{HgU}$ at Entry, Year 2 and Year 7 of the clinical trial, as well as the calculated maximum and cumulative values for both sexes at Year 7. Minimum and maximum urinary mercury concentrations for each entry are also presented. The change in number of total subjects between Entry and Year 7 reflects the overall 14\% loss to follow-up over the course of the clinical trial (DeRouen et al. 2006). The frequencies of the 
homozygous common (wildtype), heterozygous, and homozygous mutant alleles for boys and girls for CPOX4 and CPOX5 are also presented.

\subsection{CPOX4 Analyses: Effects on Acute Hg Exposure}

Results of analyses of modification by the CPOX4 variant on neurobehavioral test outcomes associated with acute $\mathrm{Hg}$ exposure (based on $\mathrm{HgU}$ at Year 2) are presented in Table 3. Among boys, significant interaction effects between CPOX4 and $\mathrm{Hg}$ exposure were observed for 3 of the 23 tests of neurobehavioral function evaluated during the clinical trial. These included the Stroop color and word/color tests, both within the Attention domain, and dominant hand finger tapping, a test of Motor function. The significant dose-response relationships between performance and acute $\mathrm{Hg}$ exposure for these tests were all in the expected (adverse) direction and were restricted to boys with the CPOX4 variant. There were no main effects observed for any of the other tests.

Among girls, CPOX4 significantly modified the effects of acute Hg exposure on only 2 tests of neurobehavioral function, the RAVALT Trial 5 and Trail 8-List 20', both within the Learning and Memory domain (Table 3). In both cases, significantly impaired performance associated with acute $\mathrm{Hg}$ exposure was observed only among girls with the CPOX4 variant. Also among girls, 4 tests were observed to have significant main effects associated with acute $\mathrm{Hg}$ exposure (not shown). Among these, only the Trails A test ( $\mathrm{p} \leq 0.03$ ) was in the impaired direction, while 3 tests, Stroop Color/Word ( $p \leq 0.03$ ), RAVALT Trial 6 ( $p \leq 0.02$ ) and the Peg test for the dominant hand ( $\mathrm{p} \leq 0.03$ ), had significant associations in the direction of improved performance. In addition, 2 tests, finger tapping dominant ( $p \leq 0.001$ ) and non-dominant ( $\mathrm{p} \leq 0.01$ ), had significantly improved performance among girls identified as having the CPOX4 variant. Aside from Motor function, no domain of neurobehavioral function was found to have more than 1 test that achieved statistical significance in terms of either main effect of acute $\mathrm{Hg}$ exposure or CPOX4 gene status among girls. Therefore, no domain could be said to be predominantly affected by acute $\mathrm{Hg}$ exposure or CPOX4 variant status among girls.

\subsection{CPOX4 Analyses: Effects on Chronic Hg Exposure among Boys}

In contrast to effects with acute $\mathrm{Hg}$ exposure, analysis of the effects of CPOX4 on neurobehavioral performance associated with chronic $\mathrm{Hg}$ exposure among boys showed numerous significant interaction terms across a wide range of tests and performance domains (Attention, Visual-Spatial, Executive Function, Learning \& Memory, and Motor). These results are presented in Table 4 . When cumulative exposure was used as the chronic $\mathrm{Hg}$ exposure measure, significant $\mathrm{Hg}$ dose-response effects were observed on 11 of 23 neurobehavioral test outcomes among boys with the CPOX4 variant, all in the impaired direction, with 7 associations being significant at $\mathrm{p} \leq 0.01$. In contrast, no significant cumulative $\mathrm{Hg}$ dose-response effects on neurobehavioral test performance were observed among boys genotyped as CPOX wildtype.

Using maximum exposure as the chronic $\mathrm{Hg}$ exposure matrix, 14 tests demonstrated significant dose-response relationships among boys with CPOX4 variant status, 12 being significant at $\mathrm{p} \leq 0.01$. The $15^{\text {th }}$ test (WRAVMA Pegs Non-Dominant) reached near significance ( $\mathrm{p} \leq 0.06)$. All of these effects are significantly adversely affected in relation to $\mathrm{Hg}$ exposure among boys with CPOX4 variant status. In contrast, among boys genotyped as CPOX wildtype, only 1 neurobehavioral function (Finger tapping-Dominant) was significantly associated with maximum $\mathrm{Hg}$ exposure ( $\mathrm{p} \leq 0.03$ ), and this was in the direction of improved performance (Table 4). 
Table 5 presents the significant main effect relationships for tests of neurobehavioral performance for which significant interaction terms were not observed among boys in the present analyses. When evaluated in terms of the cumulative chronic $\mathrm{Hg}$ exposure matrix, 4 tests of neurobehavioral performance were significantly associated with $\mathrm{Hg}$ exposure and 1 specifically with CPOX4 gene status. While all of these associations were in the direction of impaired performance, most were of only borderline significance in terms of suggesting independent effects of either chronic $\mathrm{Hg}$ exposure or CPOX4 gene status on neurobehavioral performance. When evaluated in terms of the maximum chronic $\mathrm{Hg}$ exposure matrix, 3 test outcomes were found to be significantly associated with $\mathrm{Hg}$ exposure, but none specifically with the CPOX4 variant.

\subsection{CPOX4 Analyses: Effects on Chronic Hg Exposure among Girls}

In contrast to findings among boys, no significant dose-response relationships for $\mathrm{Hg}$ exposure were observed for either maximum or cumulative chronic $\mathrm{Hg}$ exposure measures when evaluated separately among CPOX wildtype or CPOX4 variant girls, despite the fact that 2 tests (WAIS-III Digit Span and RAVLT Trial 6 - List B) had significant interaction terms between CPOX4 and maximum chronic exposure. Main effects analyses among all girls found no significant associations with either measure of chronic $\mathrm{Hg}$ exposure. However, 2 tests (finger tapping dominant and simple reaction time) had significant ( $\mathrm{p} \leq$ 0.01 or $\mathrm{p} \leq 0.02$ ) associations with CPOX 4 for both chronic $\mathrm{Hg}$ measures. Three of these 4 associations showed significantly improved performance among girls identified as having the CPOX4 variant versus those with CPOX wildtype.

\subsection{Analyses of CPOX4 Effects by Neurobehavioral Domain}

3.4.1 Attention-The Attention domain includes 3 Stroop subtests, the Digit and Spatial Span tests (considered to be more related to Attention and rehearsal than to Memory), and the Trails A test. One of the Stroop tests, the Color/Word test, introduces discordance between the color spelled out and the color in which the word is written. This test requires what may be called "directed attention", in that it includes areas of brain function other than those specifically associated with attention, and thus is not as direct a measure of simple attention as the other tests.

Among the CPOX4 acute effects analyses (Table 3), 2 of the Stroop subtests (Color and Color/Word) had significant dose-response associations among boys with the CPOX4 gene variant. The CPOX4 chronic effects analyses (Table 4) involved all of the Attention Domain tests except for the Stroop Color/Word sub-test. These analyses suggest that Attention is highly impacted by $\mathrm{Hg}$ among boys with CPOX4 variant status when evaluated in terms of either maximum or cumulative $\mathrm{Hg}$ exposure.

3.4.2. Visual-Spatial Acuity-The Visual-Spatial domain tests include Simple Reaction Time (responding to a visual stimulus), Digit Symbol (coding a symbol with a digit), and Symbol Search (scanning to find a target symbols). As shown in Table 4, significant associations for tests of Visual-Spatial acuity were found for analyses employing measures of chronic but not acute $\mathrm{Hg}$ exposure among boys. Two tests, Simple Reaction Time and Digit Symbol, had significant dose-response associations with both maximum and cumulative chronic exposure matrices among boys genotyped as having CPOX4 variant but not CPOX wildtype status. Additionally, in the main effects analyses (Table 5), the Symbol Search test had significant associations with both chronic $\mathrm{Hg}$ exposure matrices. These analyses show that the Visual-Spatial domain can be impacted by $\mathrm{Hg}$ exposures within this genetic subgroup. 
3.4.3. Executive Function-Executive Function tests included Wisconsin Card Sort (sorting cards by changing criteria) and Adult Trails B (following a trail of alternating numbers and alphabet letters). As shown in Table 4, CPOX4 was found to significantly modify the effect of $\mathrm{Hg}$ exposure on only the latter test of Executive Function among boys, and this effect was restricted to the analysis employing the maximum exposure matrix as a measure of chronic $\mathrm{Hg}$ exposure. This effect, however, was in the direction of impaired performance and the association was highly significant ( $\mathrm{p} \leq 0.003$ )

3.4.4. Learning \& Memory-The Learning \& Memory domain included 9 measures consisting of 5 sub-tests of the RAVLT (an auditory verbal learning test using a list of 15 words with distraction and delayed recall), 2 Visual Reproductions tests (redrawing a figure immediately and delayed), and the CVMT test (recalling which visual objects are repeated). Among the analyses assessing the effects of CPOX4 variant status on acute $\mathrm{Hg}$ exposure effects (Table 3), CPOX4 was found to significantly modify the adverse effects of acute $\mathrm{Hg}$ exposure on 2 of the RAVALT sub-tests (Trails 5 and Trials 8 - list A 20') among girls, constituting the principal positive observation regarding the interaction of CPOX4 and $\mathrm{Hg}$ exposure among girls in this study. No associations of gene status with indices of chronic $\mathrm{Hg}$ exposure among girls were observed.

In contrast, 3 RAVALT sub-tests were found to have significant dose-response associations with the maximum $\mathrm{Hg}$ chronic exposure matrix among boys with the CPOX4 variant (Table 4), and 1 also having a significant association with the cumulative $\mathrm{Hg}$ exposure matrix. In the main effects analyses (Table 5), both immediate and delayed Visual Reproductions tests were found to have significant independent associations with both chronic $\mathrm{Hg}$ exposure matrices.

3.4.5. Motor Function-The Motor function domain tests include Finger Tapping (number of taps in a fixed time) and the Pegs test (number of dowels inserted into a hole in a fixed time). Both tests evaluate dominant and non-dominant hand performance separately. All 4 of these tests either approached significance or were significantly associated with the maximum exposure matrix among boys carrying the CPOX4 variant, with 1 association in the direction of improved performance among boys genotyped as CPOX wildtype (Table 4). There were 3 tests (excluding Peg dominant hand) related to the cumulative $\mathrm{Hg}$ exposure matrix among boys with the CPOX4 variant. Whereas most of the associations observed in this domain demonstrated only moderate significance levels, the interaction of CPOX4 and $\mathrm{Hg}$ exposure on the Finger Tapping non-dominant hand test was highly significant.

\subsection{CPOX5 Analyses}

No significant interaction effects were observed for CPOX5 and any measure of $\mathrm{Hg}$ exposure among either boys or girls. Moreover, no main effects were observed for CPOX5.

\subsection{Summary of Results}

CPOX4 gene status was found to modify the adverse effects of chronic $\mathrm{Hg}$ exposure on a wide range of neurobehavioral performance test results among boys. All of these effects were in the direction of impaired performance and spanned all 5 neurobehavioral domains. Many of the observed associations were highly significant ( $\mathrm{s} \leq 0.01)$. These highly consistent observed associations, affecting more than half the neurobehavioral tests evaluated, cannot be dismissed due to the large numbers of tests and exposures evaluated. If we assume that the outcomes for each of the 23 tests evaluated were totally independent of each other, the binomial probabilities of our findings for the two chronic measures would be $<2 \times 10^{-12}$ for cumulative exposure and $<2 \times 10^{-17}$ for maximum exposures. While there 
clearly are strong correlations between performances on various behavioral tests, the consistency of these results is nonetheless highly compelling.

Finally, the converse lack of associations between $\mathrm{Hg}$ exposure and CPOX wildtype status suggests that the CPOX4 variant may be important in mediating neurotoxic effects of $\mathrm{Hg}$ exposure in boys. The absence of associations when comparable analyses were conducted for the CPOX5 variant argues against these observations being due to some selection process.

\section{Discussion}

Numerous studies have proposed a component of genetic susceptibility to neurobehavioral disorders associated with mercury and other xenobiotic exposures (Braun et al. 2006; Gundacker et al. 2010; Engström et al. 2008; Suk and Collman 1998), although the modifying effects of commonly expressed genetic variants on these associations are just beginning to be defined. This is the first study, to our knowledge, to describe a genetic polymorphism that modifies the effects of mercury exposure on a wide variety of neurobehavioral functions in children. Previous studies provided evidence of significant associations between $\mathrm{Hg}$ exposure and the CPOX4 variant on neurobehavioral functions in adult dental professionals (Echeverria et al. 2006), although observed joint effects in that study were found to be strictly additive in nature. The present findings of synergistic, i.e., more than additive, interactions between $\mathrm{Hg}$ and CPOX4 on numerous neurobehavioral functions are consistent with potentially heightened susceptibility of children to the adverse neurobehavioral effects of $\mathrm{Hg}$ specifically associated with the CPOX4 genetic variant.

The paucity of findings of independent effects of $\mathrm{Hg}$ exposure on tests of neurobehavioral function in this study provide some consistency with findings from the dental amalgam clinical trial (DeRouen et al. 2006), in which exposure to $\mathrm{Hg}$ from dental amalgam was found not to be associated with deficits in any tests of neurobehavioral performance among either boys or girls. However, when controlling for CPOX gene status as performed here, $\mathrm{Hg}$ exposure was strongly associated with diminished performance across a wide range of the same tests, among boys with the CPOX4 variant. Diminished performance was most predominantly observed in tests of Attention, suggesting possible impairment of attentional vitality and flexibility, e.g., ability to sustain attention or to shift between 2 sequences held in working memory (Echeverria et al., 2002). Significant interactions between $\mathrm{Hg}$ exposure and CPOX4 on tests of Learning \& Memory and of Visual-Spatial acuity were also observed, suggesting possible decrements of verbal learning and memory as well as of perceptual cognition. Effects on tests of Motor function, including measures of manual coordination and fine motor speed, also appear to be adversely affected when evaluated within the context of chronic $\mathrm{Hg}$ exposure among boys with the CPOX4 variant. These findings have important public health implications, inasmuch as mean urinary mercury levels among boys in this study ranged from $1.4(1.3-1.6) \mu \mathrm{g} / \mathrm{g}$ creatinine at baseline to a maximum of $2.2(1.8-2.5) \mu \mathrm{g} / \mathrm{g}$ creatinine at Year 2 of follow-up in the dental amalgam clinical trial. By comparison, geometric mean urinary mercury levels measured among a nationally representative sample of children 12-19 years of age acquired as part of the 2003-2004 U.S. National Health and Nutrition Examination Survey (Centers for Disease Control and Prevention 2007) were $0.358(0.313-0.408) \mu \mathrm{g} / \mathrm{g}$ creatinine. Although this value is substantially lower than those measured in the present study, the mean urinary $\mathrm{Hg}$ concentration in the $90^{\text {th }}$ percentile of that sample was $1.59(1.13-2.52) \mu \mathrm{g} / \mathrm{g}$ creatinine, comparable to the range of $\mathrm{Hg}$ concentrations at which adverse neurologic effects of $\mathrm{Hg}$ were observed herein among boys with CPOX4. These observations suggest potential adverse neurobehavioral effects of $\mathrm{Hg}$ among boys with the CPOX4 variant who fall within the top $10 \%$ of subjects sampled within that survey for $\mathrm{Hg}$ exposure. 
The mechanistic association of CPOX4 to neurobehavioral functions remains to be delineated, although potential alterations in physiological heme availability and/or hemedependent processes associated with diminished CPOX4 activity may underlie this effect (Li and Woods, 2009). In this regard, heme is known to play a critical role as a signaling molecule in glutaminergic neuronal receptor processing and synapse development (Chernova et al. 2006; Sengupta et al. 2005), as well as in the regulation of serotonin (5hydroxytryptamine) synthesis and signaling in the central nervous system (Litman and Correia 1983, 1985). Disorders of both systems have been implicated as etiologic in a variety of neurodevelopmental and neurobehavioral disorders (Chernova et al. 2011; Chugani et al. 1999; Smith et al. 2012), and both could be amenable to disruption by heme deficiency during critical periods of neurological development in children, particularly in association with mercury exposure ( $\mathrm{Li}$ and Woods 2009). While these observations provide a scientific rationale for the diminished neurobehavioral performance observed here among boys with the CPOX4 variant and $\mathrm{Hg}$ exposure, further studies are required to define the specific mechanistic events underlying this association.

The absence of effects of CPOX5 on neurobehavioral functions when evaluated in relation to any measure of $\mathrm{Hg}^{0}$ exposure in this study suggests that the CPOX4 variant may act in a genotype-selective manner to mediate the adverse neurobehavioral effects of $\mathrm{Hg}$ exposure observed here. While the potential effects of CPOX5 on CPOX enzymatic activity, heme bioavailability, or processes affecting neurological function are not known, CPOX5 need not be viewed as incapable of affecting biological processes, inasmuch as synonymous SNPs are widely recognized as mediating changes in translation kinetics, protein folding and other factors that underlie a wide variety of neurological and other disorders in humans (Chamary et al. 2006; Duan et al. 2003; Komar 2007). Moreover, the heterozygous and full mutant variants of CPOX5 were distributed quite differentially from those of CPOX4 within this cohort, only 7 subjects (2\%) sharing both CPOX4 and CPOX5 variant status, militating against selection bias in terms of findings observed with respect to those with CPOX4. Further research analyzing multiple SNPs within the CPOX gene as well as others associated with heme-dependent neurotransmitter processing pathways is required to identify the mechanisms underlying the apparent selective effects of CPOX4 seen here.

Notable differences between boys and girls in the effects of $\mathrm{Hg}$ exposure and the CPOX4 variant on neurobehavioral test performance were observed in this study. Although $\mathrm{Hg}$ exposure from dental amalgam was comparable among boys and girls participating in the clinical trial (DeRouen et al, 2006), sex-related differences in $\mathrm{Hg}$ toxicokinetics that may afford greater $\mathrm{Hg}$ excretion and, consequently, lesser likelihood of $\mathrm{Hg}$ retention and accumulation in girls than boys may contribute to this effect (Woods et al. 2007). Numerous other factors that include genetic and hormonal differences affecting brain development, structure and function between boys and girls are also likely to contribute to the gender differences observed here (Gochfeld 2007; Hines et al. 2010; Vahter et al. 2007a,b; Valentino et al. 2012). Differences in detection sensitivity for CPOX4 between boys and girls in this study have a less clear explanation, although genetic factors underlying gender differences in numerous psychiatric and neurobehavioral disorders have been reported (Baca-Garcia et al. 2002; Gaub and Carlson 1997; Harrison and Tunbridge 2008; Samochowiec et al. 2004). The observation that neither $\mathrm{Hg}$ exposure nor CPOX4 alone substantially affected neurobehavioral performance in girls suggests that sex-related predisposition, in addition to differences in $\mathrm{Hg}$ toxicokinetics, affects susceptibility. Of note, measures of cognitive function and other behaviors not specifically related to reproduction are often sex-linked, accounting for substantial differences in response to many chemical agents, with subsequent expression in behavior (Weiss 2002). In this respect, many classes of chemicals including dioxins and polychlorinated biphenyls (Weiss 2002), metals (nickel arsenic, lead, cadmium, mercury) (Vahter et al. 2007a,b), pesticides (paraquat, 
dithiocarbamate, triazole fungicides) (Vahter et al. 2007a), cigarette smoke (Kelada et al 2002); and various classes of drugs (Calabrese 1985) are reported to differentially affect neurologic functions in males and females, both in humans and animal models (Vahter et al. 2007a; Bjorklund et al., 2007; Gochfeld 2007). The sexually divergent responses to $\mathrm{Hg}$ exposure and genetic disposition observed in the present study highlight the importance of considering such differences in development of strategies aimed at risk assessment and prevention, especially in children.

In conclusion, the present studies demonstrate significant adverse effects on neurobehavioral functions associated with chronic $\mathrm{Hg}$ exposure and the CPOX4 genetic variant among children, with effects manifested predominantly among boys. These findings are the first to describe a genetic polymorphism that modifies the effects of $\mathrm{Hg}$ exposure on neurobehavioral functions in children, and suggest directions for future research to define mechanisms underlying differential sensitivity to mercury between boys and girls.

\section{Acknowledgments}

This research was funded by grants P42ES04696, P30ES07033 and R21ES019632 to the University of Washington from the National Institute of Environmental Health Sciences (NIEHS) of the National Institutes of Health. Additional funding was provided by the Wallace Research Foundation. We thank Professor and Dean Emerita Nancy Fugate Woods, Department of Family and Child Nursing, University of Washington for insightful discussions regarding the analysis and interpretation of the findings presented in this manuscript.

\section{Abbreviations}

$\begin{array}{ll}\text { Hg } & \text { mercury } \\ \text { IQ } & \text { intelligence quotient } \\ \text { CPOX } & \text { coproporphyrinogen oxidase }\end{array}$

\section{References}

Baca-García E, Vaquero C, Diaz-Sastre C, Saiz-Ruiz J, Fernández-Piqueras J, de Leon J. A genderspecific association between the serotonin transporter gene and suicide attempts. Neuropsychopharmacol. 2002; 26:692-695.

Björklund O, Kahlström J, Salmi P, Ove Ögren S, Vahter M, Chen J-C, et al. The effects of methylmercury on motor activity are sex- and age-dependent, and modulated by genetic deletion of adenosine receptors and caffeine administration. Toxicol. 2007; 241:119-133.

Braun JM, Kahn RS, Froehlich T, Auinger P, Lanphear BP. Exposures to environmental toxicants and attention deficit hyperactivity disorder in U.S. children. Environ Health Perspect. 2006; 114:19041909. [PubMed: 17185283]

Calabrese, EJ. Toxic Susceptibility: Male/Female Differences. New York: Wiley-Interscience; 1985.

Centers for Disease Control and Prevention. [15 May 2012] National Health and Nutrition Examination Survey. 2007. Availabile: http://www.cdc.gov/nchs/nhanes.htm

Chamary JV, Parmley JL, Hurst LD. Hearing silence: non-neutral evolution at synonymous sites in mammals. Nat Rev Genet. 2006; 7:98-108. [PubMed: 16418745]

Chernova T, Steinhert JR, Richards P, Mistry R, Challiss RAJ, Jukes-Jones R, et al. Early failure of Nmethyl-D-aspartate receptors and deficient spine formation induced by reduction of regulatory heme in neurons. Molec Pharmacol. 2011; 79:844-854. [PubMed: 21325018]

Chernova T, Nicotera P, Smith AG. Heme deficiency is associated with senescence and causes suppression of $\mathrm{N}$-methyl-D-aspartate receptor subunits expression in primary cortical neurons. Molec Pharmacol. 2006; 69:697-705. [PubMed: 16306232] 
Chugani DC, Musik O, Behen M, Rothermel R, Janisse JJ, Lee J, Chugani HT. Developmental changes in brain serotonin synthesis capacity in autistic and nonautistic children. Ann Neurol. 1999; 45:287-295. [PubMed: 10072042]

Clarkson TW. Three modern faces of mercury. Environ Health Perspect. 2003; 110:11-23. [PubMed: 11834460]

Counter SA, Buchanan LH. Mercury exposure in children: a review. Toxicol Appl Pharmacol. 2004; 198:209-230. [PubMed: 15236954]

Davidson PW, Myers GJ, Weiss B. Mercury exposure and child development outcomes. Pediatrics. 2004; 113(suppl 4):1023-1029. [PubMed: 15060195]

DeRouen TA, Leroux BG, Martin MD, Townes BD, Woods JS, Leitão J, Castro-Caldas A, Braveman $\mathrm{N}$. Issues in the design and analysis of a randomized clinical trial to assess the safety of dental amalgam fillings in children. Contr Clin Trials. 2002; 23:301-320.

DeRouen TA, Martin MD, Leroux BG, Townes BD, Woods JS, Leitão J, Castro-Caldas A, Luis H, Bernardo M, Rosenbaum G, Martins IP. Neurobehavioral effects of dental amalgam in children: a randomized clinical trial. JAMA. 2006; 295(15):1784-1792. [PubMed: 16622140]

Duan J, Wainwright MS, Comeron JM, Saitou N, Sanders AR, Gelernter J, et al. Synonymous mutations in the human dopamine receptor D2 (DRD2) affect mRNA stability and synthesis of the receptor. Hum Molec Genet. 2003; 112:205-216. [PubMed: 12554675]

Echeverria D, Aposhian HV, Woods JS, Heyer NJ, Aposhian MM, Bittner AC, et al. Neurobehavioral effects from exposure to dental amalgam $\mathrm{Hg}^{0}$ : New distinctions between recent exposure and $\mathrm{Hg}$ body burden. FASEB J. 1998; 12:971-980. [PubMed: 9707169]

Echeverria D, Heyer NJ, Bittner AC, Rohlman D, Woods JS. Test-retest reliability and factor stability of the Behavioral Evaluation for Epidemiology Studies test battery. Percept Motor Skills. 2002; 95:845-867. [PubMed: 12509185]

Echeverria D, Woods JS, Heyer NJ, Martin MD, Rholman D, Farin FM, et al. The association between serotonin transporter gene promoter polymorphism (5-HTTLPR) and elemental mercury exposure on mood and behavior in humans. J Toxicol Environ Health. 2010; 73:552-569.

Echeverria D, Woods JS, Heyer NJ, Rholman D, Farin FM, Bittner AC Jr, et al. Chronic low-level mercury exposure, BDNF polymorphism, and associations with memory, attention and motor function. Neurotox Teratol. 2005; 27:781-796.

Echeverria D, Woods JS, Heyer NJ, Rholman D, Farin FM, Li T, et al. The association between a genetic polymorphism of coproporphyrinogen oxidase, dental mercury exposure, and neurobehavioral response in humans. Neurotox Teratol. 2006; 28:39-48.

Engström KS, Strömberg U, Lundh T, Jonhansson I, Vessby B, Hallmans G, et al. Genetic variation in glutathione-related genes and body burden of methylmercury. Environ Health Perspect. 2008; 116:734-739. [PubMed: 18560528]

Evens CC, Martin MD, Woods JS, Soares HL, Bernardo M, Leitão J, et al. Examination of dietary methylmercury exposure in the Casa Pia study of the health effects of dental amalgams in children. J. Toxicol Environ Health. 2001; 64:521-530.

Faustman EM, Silbernagel SM, Fenske RA, Burbacher TM, Ponce RA. Mechanisms underlying children's susceptibility to environmental toxicants. Environ Health Perspect. 2000; 108(Suppl 1): 13-21. [PubMed: 10698720]

Gaub M, Carlson CL. Gender differences in ADHD: A meta-analysis and critical review. J Amer Acad Child Adoles Psychiat. 1997; 36:1036-1045.

Gochfeld M. Framework for gender differences in human and animal toxicology. Environ Res. 2007; 104:4-21. [PubMed: 16616135]

Goering PL, Galloway WD, Clarkson TW, Lorscheider FL, Berlin M, Rowland AS. Toxicity assessment of mercury vapor from dental amalgams. Fundam Appl Toxicol. 1992; 19:319-329. [PubMed: 1360929]

Gundacker C, Gencik M, Hengstschläger M. The relevance of individual genetic background for the toxicokinetics of two significant neurodevelopmental toxicants: mercury and lead. Mut Res. 2010; 705:130-140. [PubMed: 20601101] 
Harrison PJ, Tunbridge EM. Catechol- $O$-methyltransferase (COMT): A gene contributing to sex differences in brain function, and to sexual dimorphism in the predisposition to psychiatric disorders. Neuropsychopharmacol. 2008; 33:3037-3045.

Heyer NJ, Echeverria D, Bittner AC, Farin FM, Garabedian CC, Woods JS. Chronic low-level mercury exposure, BDNF polymorphism and associations with self-reported symptoms and mood. Toxicol Sci. 2004; 81:354-363. [PubMed: 15254338]

Heyer NJ, Echeverria D, Farin FM, Woods JS. The association between serotonin transporter gene promoter polymorphism (5-HTTLPR), self-reported symptoms, and dental mercury exposure. J Toxicol Environ Health. 2008; 71:1318-1326.

Heyer NJ, Echeverria D, Martin MD, Farin FM, Woods JS. Catechol- $O$-methyltransferase (COMT) Val158Met functional polymorphism, dental mercury exposure, and self-reported symptoms and mood. J Toxicol Environ Health. 2009; 72:599-609.

Hines RN, Sargent D, Autrup H, Birnbaum LS, Brent RL, Doerrer NG, et al. Approaches for assessing risk to sensitive populations: Lessons learned from evaluating risk in the pediatric population. Toxicol Sci. 2010; 113:4-26. [PubMed: 19770482]

Kelada SN, Costa-Mallen P, Costa LG, Smith-Weller T, Franklin GM, Swanson PD, et al. Gender difference in the interaction of smoking and monoamine oxidase B intron 13 genotype in Parkinson's disease. Neurotoxicol. 2002; 23:515-519.

Komar AA. SNPs, silent but not invisible. Science. 2007; 315:466-467. [PubMed: 17185559]

Landrigan PJ, Goldman LR. Children's vulnerability to toxic chemicals: A challenge and opportunity to strengthen health and environmental policy. Health Affairs. 2011; 30:842-850. [PubMed: 21543423]

Levy M, Schwartz S, Dijak M, Weber J-P, Tardif R, Rouah F. Childhood urine mercury excretion: dental amalgam and fish consumption as exposure factors. Environ Res. 2004; 94:283-290. [PubMed: 15016596]

Li T, Woods JS. Cloning, expression, and biochemical properties of CPOX4, a genetic variant of coproporphyrinogen oxidase that affects susceptibility to mercury toxicity in humans. Toxicol Sci. 2009; 109:228-236. [PubMed: 19339664]

Litman DA, Correia MA. L-Tryptophan: A common denominator of biochemical and neurological events of acute hepatic porphyria? Science. 1983; 222:1031-1033. [PubMed: 6648517]

Litman DA, Correia MA. Elevated brain tryptophan and enhanced 5-hydroxytryptamine turnover in acute hepatic heme deficiency: clinical implications. J Pharmacol Exper Therap. 1985; 232:337345. [PubMed: 3968635]

Martins IP, Castro-Caldas A, Townes B, Ferreira G, Rodrigues P, Marques S, et al. Age and sex difference in neurobehavioral performance: a study of Portuguese elementary school children. Int $\mathbf{J}$ Neurosci. 2005; 115:1687-1709. [PubMed: 16287634]

Pingree SD, Simmonds PL, Woods JS. Effects of 2,3-dimercapto-1-propanesulfonic acid (DMPS) on tissue and urine mercury levels following prolonged methylmercury exposure in rats. Toxicol Sci. 2001; 61:224-233. [PubMed: 11353131]

Samochowiec J, Hajduc A, Samochowiec A, Horodnicki J, Stepien G, Grzywacz A, et al. Association of MAO-A, COMT, and 5-HTT genes polymorphisms in patients with anxiety disorders of the phobic spectrum. Psychiat Res. 2004; 128:21-26.

Sengupta A, Hon T, Zhang L. Heme deficiency suppresses the expression of key neuronal genes and causes neuronal cell death. Brain Res Molec Brain Res. 2005; 137:23-30. [PubMed: 15950757]

Slade PD, Townes BD, Rosenbaum G, Martins IP, Luis H, Bernardo M, et al. The serial use of child neurocognitive tests: development versus practice effects. Psychol Assess. 2008; 20:361-369. [PubMed: 19086759]

Smith AG, Raven EL, Chernova T. The regulatory role of heme in neurons. Metallomics. 2012; 3:955962. [PubMed: 21922110]

Suk WA, Collman GW. Genes and the environment: Their impact on children's health. Environ Health Perspect. 1998; 106(suppl 3):817-820. [PubMed: 9646043]

Townes BD, Rosenbaum G, Martin MD, Martins IP, Luis H, Bernardo M. A longitudinal factor analytic study of children's neurocognitive abilities. Int J Neurosci. 2008a; 118(7):1009-1023. [PubMed: 18569157] 
Townes BD, Martins IP, Castro-Caldas A, Rosenbaum G, DeRouen T. Repeat test scores on neurobehavioral measures over an eight-year period in a sample of Portuguese children. Int $\mathbf{J}$ Neurosci. 2008b; 118(1):79-93. [PubMed: 18041607]

Vahter M, Åkesson A, Lidén C, Ceccatelli S, Berglund M. Genetic differences in the disposition and toxicity of metals. Environ Res. 2007a; 104:85-95. [PubMed: 16996054]

Vahter M, Gochfeld M, Casati B, Thiruchelvam M, Falk-Filippson A, Kavlock R, et al. Implications of gender differences for human health risk assessment and toxicology. Environ Res. 2007b; 104:7084. [PubMed: 17098226]

Valentino RJ, Reyes B, Bockstaele EV, Bangasser D. Molecular and cellular sex differences at the intersection of stress and arousal. Neuropharmacol. 2012; 62:13-20.

Weiss B. Sexually dimorphic nonreproductive behaviors as indicators of endocrine disruption. Environ Health Perspect. 2002; 110(suppl 3):387-391. [PubMed: 12060833]

Woods JS, Echeverria D, Heyer NJ, Simmonds PL, Wilkerson J, Farin FM. The association between genetic polymorphisms of coproporphyrinogen oxidase and an atypical porphyrinogenic response to mercury exposure in humans. Toxicol Appl Pharmacol. 2005; 206:113-120. [PubMed: 15967199]

Woods JS, Martin MD, Leroux BG, DeRouen TA, Leitão JG, Bernardo M, et al. The contribution of dental amalgam to urinary mercury excretion in children. Environ Health Perspect. 2007; 115:527-1531. 


\section{Highlights}

- CPOX4, a SNP of coproporphyrinogen oxidase, enhances Hg neurotoxicity in children.

- Interactions of CPOX4 and $\mathrm{Hg}$ span all 5 domains of behavioral performance.

- Modification of $\mathrm{Hg}$ neurotoxicity by CPOX4 is restricted largely to boys.

- Sexually dimorphic genetic susceptibility to $\mathrm{Hg}$ toxicity in children is apparent. 
Table 1

Neurobehavioral tests assessed with mean scores for Year 7 (Final year of clinical trial)

\begin{tabular}{|c|c|c|c|}
\hline Test / Domain & Measure $^{a}$ & $\begin{array}{c}\text { Boys } N=121 \\
\text { Mean (SD) }\end{array}$ & $\begin{array}{c}\text { Girls N }=118 \\
\text { Mean (SD) }\end{array}$ \\
\hline \multicolumn{4}{|c|}{ Attention } \\
\hline Stroop Test - Color, & $\#$ correct $\uparrow$ & $66.41(11.96)$ & $69.23(10.43)$ \\
\hline Word & \# correct $\uparrow$ & $90.07(15.18)$ & $91.47(15.23)$ \\
\hline Color-Word & $\#$ correct $\uparrow$ & $41.84(9.78)$ & $43.88(8.75)$ \\
\hline WAIS-III - Digit Span & \# correct $\uparrow$ & $14.34(3.68)$ & $14.14(2.77)$ \\
\hline WMS III - Spatial Span & $\#$ correct $\uparrow$ & $15.84(3.02)$ & $15.58(3.12)$ \\
\hline Adult Trails A & Time (sec) $\downarrow$ & $26.35(10.62)$ & $30.38(11.38)$ \\
\hline \multicolumn{4}{|c|}{ Visual-Spatial } \\
\hline Simple Reaction Time & Time (sec) $\downarrow$ & $0.74(0.15)$ & $0.77(0.13)$ \\
\hline WAIS III - Digit Symbol & \# correct $\uparrow$ & $72.45(17.09)$ & $76.68(13.87)$ \\
\hline Symbol Search & \# correct $\uparrow$ & $33.15(8.85)$ & $34.57(8.04)$ \\
\hline \multicolumn{4}{|c|}{ Executive Functioning } \\
\hline Wisconsin Card Sort - Categories Completed & \# categories $\uparrow$ & $3.06(1.38)$ & $3.08(1.47)$ \\
\hline Adult Trails B & Time (sec) $\downarrow$ & $65.69(26.99)$ & $63.41(23.67)$ \\
\hline \multicolumn{4}{|c|}{ Learning \& Memory } \\
\hline RAVALT Tr1 - List A & \# correct $\uparrow$ & $5.61(1.49)$ & $6.10(1.86)$ \\
\hline Tr5 - List A & $\#$ correct $\uparrow$ & $11.24(2.20)$ & $11.54(2.23)$ \\
\hline Tr6 - List B & \# correct $\uparrow$ & $4.73(1.38)$ & $5.27(1.56)$ \\
\hline $\operatorname{Tr} 7$ - List A/Post B & \# correct $\uparrow$ & $9.85(2.56)$ & $10.22(2.48)$ \\
\hline Tr8 - List A after 20' & \# correct $\uparrow$ & $9.30(2.72)$ & $10.07(2.77)$ \\
\hline WMS-III - Visual Reproductions - Immediate & $\#$ correct $\uparrow$ & $34.72(4.65)$ & $36.61(2.98)$ \\
\hline Delayed & \# correct $\uparrow$ & $31.98(6.96)$ & $34.90(4.02)$ \\
\hline CVMT d-Prime & Score $\uparrow$ & $1.51(.94)$ & $1.61(.88)$ \\
\hline \multicolumn{4}{|c|}{ Motor } \\
\hline WRAVMA -Pegs - Dominant & \# Pegs $\uparrow$ & $47.40(8.50)$ & $49.92(6.30)$ \\
\hline Non Dominant & \# Pegs $\uparrow$ & $44.33(7.57)$ & $45.02(6.12)$ \\
\hline Finger Tapping - Dominant & \# Taps $\uparrow$ & $52.75(5.59)$ & $48.44(5.72)$ \\
\hline Non Dominant & \# Taps $\uparrow$ & $46.66(5.90)$ & $42.53(5.81)$ \\
\hline
\end{tabular}

${ }^{a}$ Arrows show direction of improved performance. 


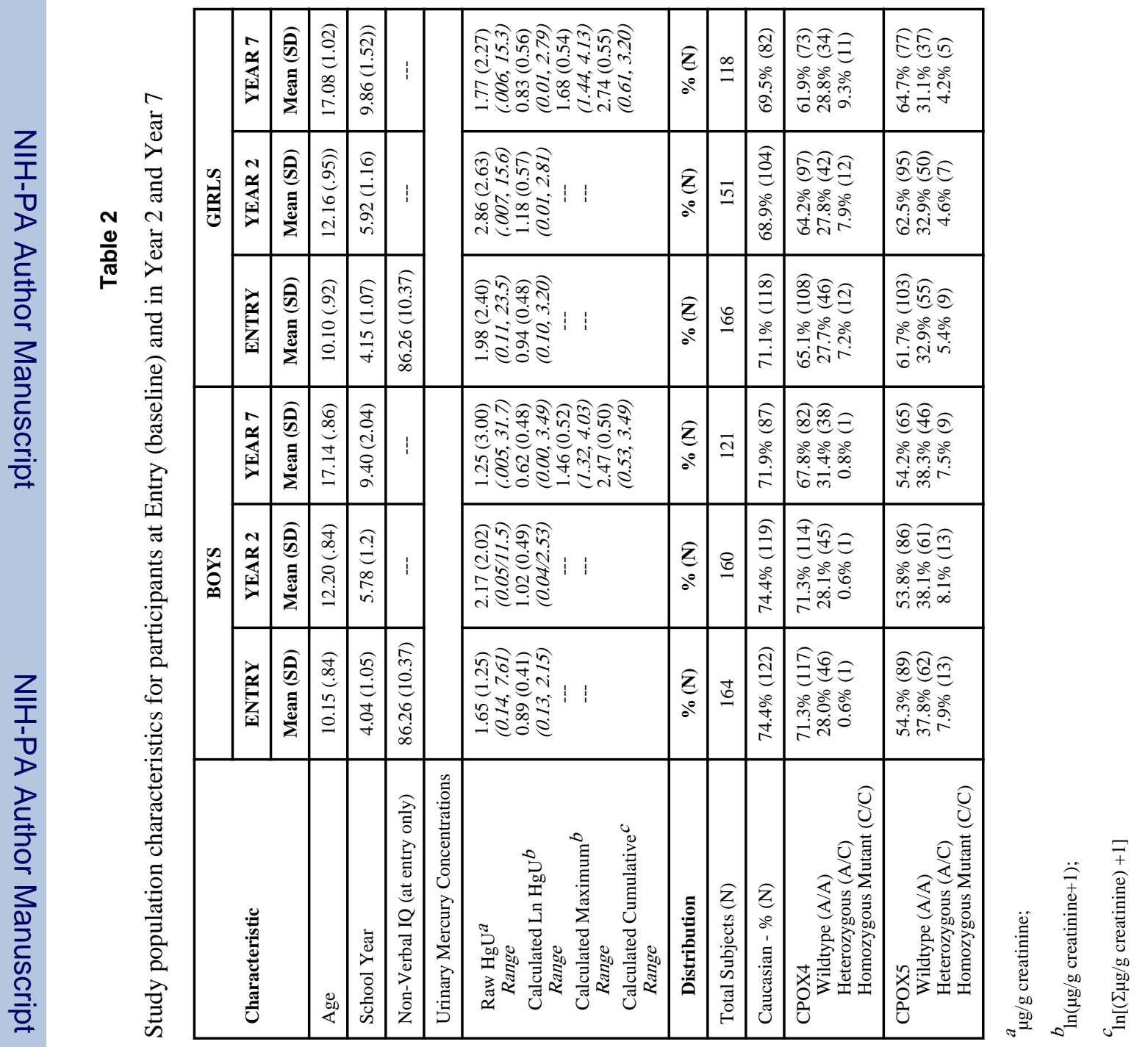


Table 3

Acute Hg dose-response effects among boys and girls with CPOX WT or CPOX4 variant in Year 2

\begin{tabular}{|c|c|c|c|c|}
\hline \multirow{2}{*}{ Measure } & \multicolumn{2}{|c|}{ WT } & \multicolumn{2}{|c|}{ Het or Mut } \\
\hline & Beta (SE) & $\mathbf{r}_{\text {part }}(\mathbf{p})$ & Beta (SE) & $\mathbf{r}_{\text {part }}(\mathbf{p})$ \\
\hline \multicolumn{5}{|c|}{ BOYS } \\
\hline \multicolumn{5}{|c|}{ Attention Domain } \\
\hline Stroop Test - Color & $-.19(1.80)$ & $-01(.92)$ & $-8.40(2.80)$ & $-.42(.005)$ \\
\hline Stroop Test - Word/Color & $1.09(1.51)$ & $.07(.47)$ & $-3.50(1.68)$ & $-.31(.04)$ \\
\hline \multicolumn{5}{|c|}{ Motor Domain } \\
\hline Finger Tapping - Dominant & $1.21(.91)$ & $.13(.19)$ & $-3.25(1.53)$ & $-.32(.04)$ \\
\hline \multicolumn{5}{|c|}{ GIRLS } \\
\hline \multicolumn{5}{|c|}{ Learning and Memory Domain } \\
\hline RAVALT TR 5 & $.28(.43)$ & $.07(.51)$ & $-1.34(.47)$ & $-.38(.006)$ \\
\hline RAVALT TR 8 - List A 20' & $.34(.45)$ & $.08(.46)$ & $-1.69(.55)$ & $-.40(.003)$ \\
\hline
\end{tabular}

Values in bold signify $\mathrm{p} \leq 0.05$. 


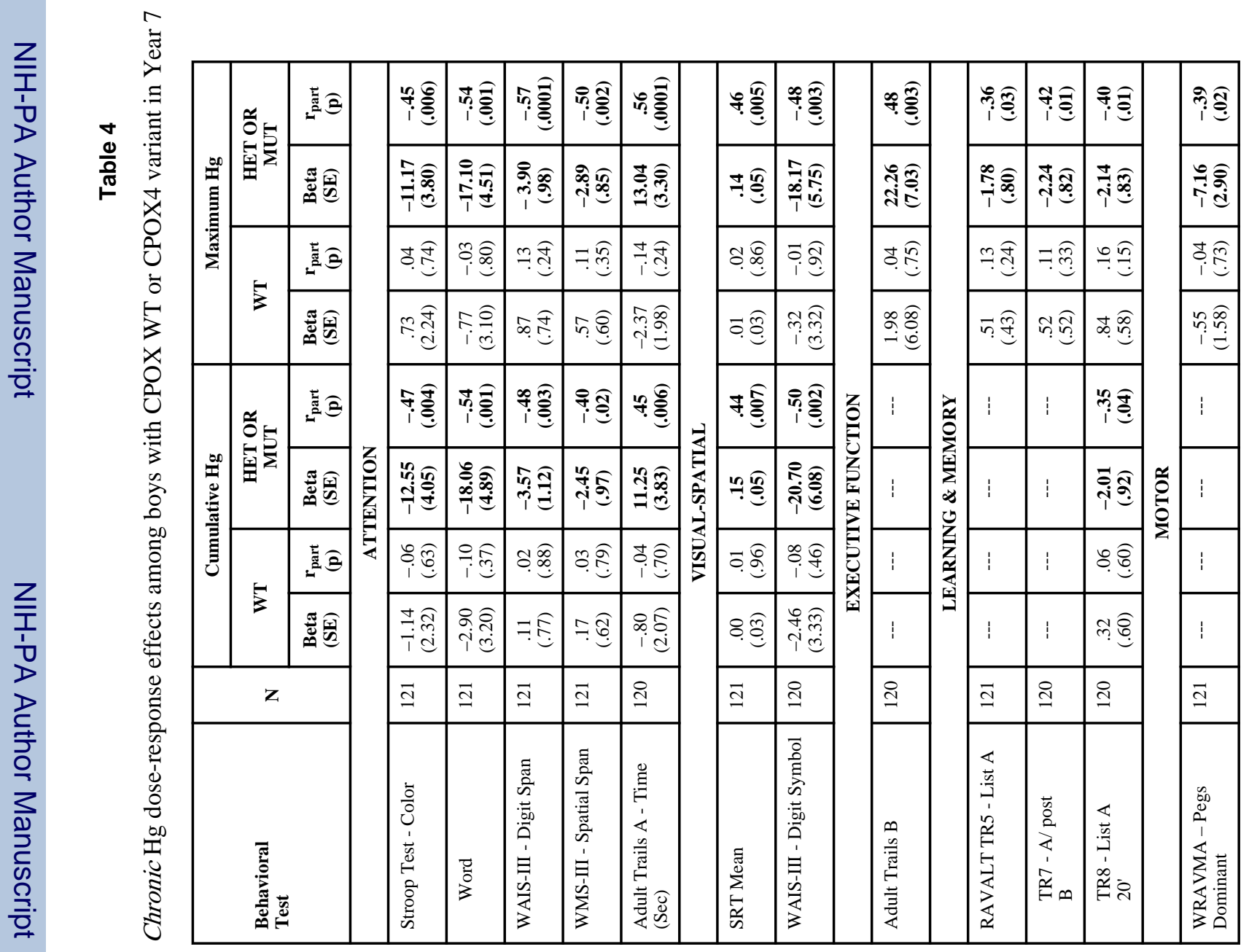




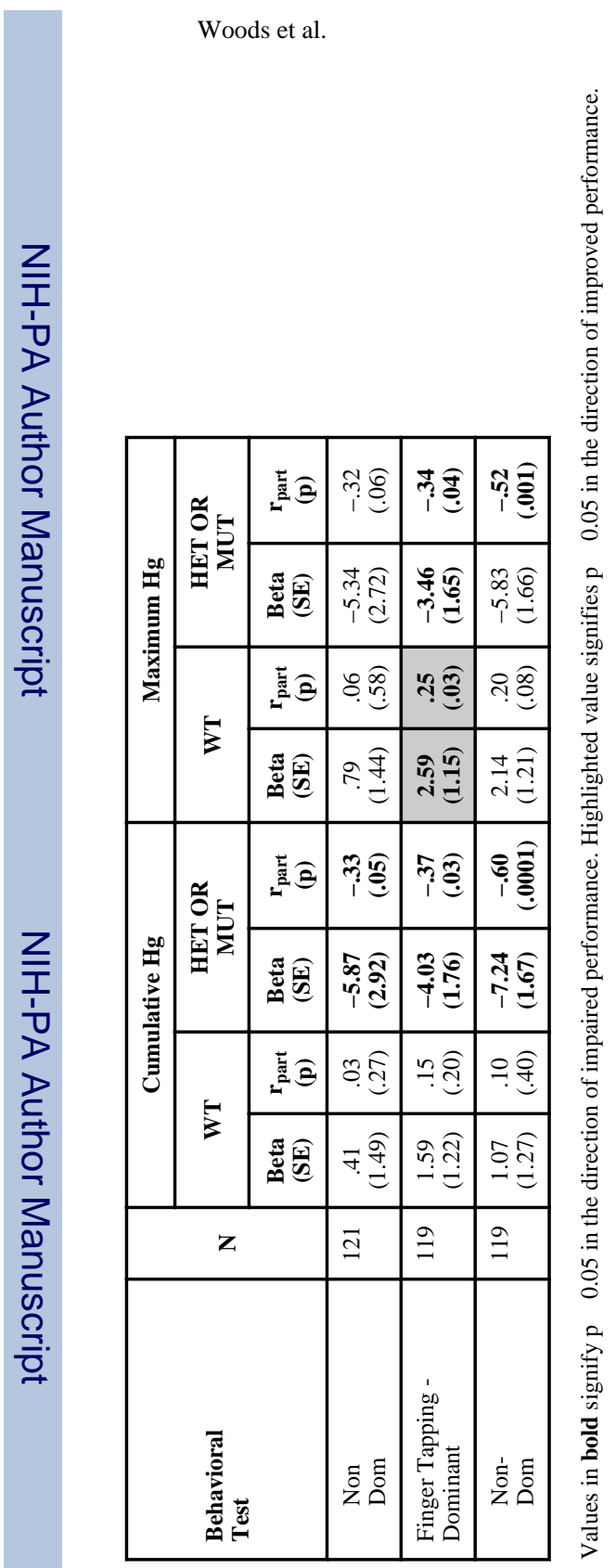

Page 20 


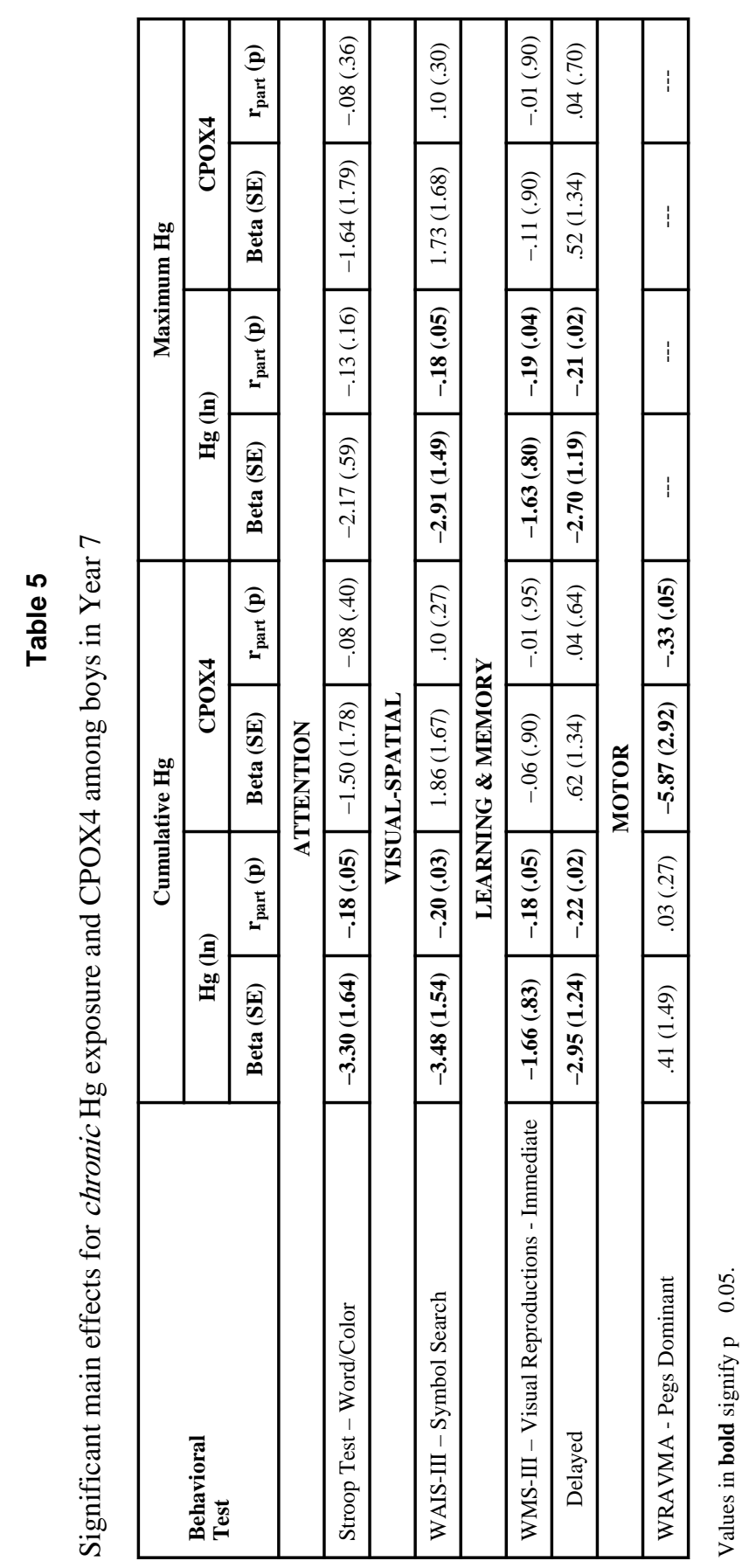

Neurotoxicol Teratol. Author manuscript; available in PMC 2013 September 01. 